\title{
The Role of Technology in COVID-19 Pandemic Management and Its Financial Impact
}

\author{
Jin Zhao, ${ }^{1}$ Zubair Ahmad $\left(\mathbb{D},{ }^{2}\right.$ Saima K. Khosa $\mathbb{D}^{3},{ }^{3}$ M. Yusuf $\mathbb{D}^{4},{ }^{4}$ Osama Abdulaziz Alamri, ${ }^{5}$ \\ and Mohamed S. Mohamed (iD) \\ ${ }^{1}$ School of Finance, Shanghai Lixin University of Accounting and Finance, Shanghai, China \\ ${ }^{2}$ Department of Statistics, Yazd University, P.O. Box 89175-741, Yazd, Iran \\ ${ }^{3}$ Department of Statistics, Bahauddin Zakariya University, Multan, Pakistan \\ ${ }^{4}$ Department of Mathematics, Faculty of Science, Helwan University, Helwan, Egypt \\ ${ }^{5}$ Department of Statistics, Faculty of Science, University of Tabuk, Tabuk, Saudi Arabia \\ ${ }^{6}$ Department of Mathematics, Faculty of Education, Ain Shams University, Cairo 11341, Egypt
}

Correspondence should be addressed to Zubair Ahmad; z.ferry21@gmail.com and Saima K. Khosa; saima.khosa@bzu.edu.pk

Received 19 April 2021; Accepted 9 August 2021; Published 13 September 2021

Academic Editor: Abdel-Haleem Abdel-Aty

Copyright ( 92021 Jin Zhao et al. This is an open access article distributed under the Creative Commons Attribution License, which permits unrestricted use, distribution, and reproduction in any medium, provided the original work is properly cited.

\begin{abstract}
A pandemic caused by the novel coronavirus (COVID-19) is causing an unprecedented situation for the world's health services. Health, local communities, and government are adversely affected by the COVID-19 pandemic. Moreover, on January 21, 2020, the WHO Emergency Committee declared a global health emergency because of increasing numbers of COVID-19 case notifications from countries overseas. While the pandemic caused grave damage to socioeconomic phenomena and multiple global phenomena such as commodity prices, remittances, trade, tourism, significant job loss, and drastically lower wages, the pandemic has also negatively affected multiple global phenomena. The advent of technology has spurred significant changes in many aspects of our lives and improved the exchange of information, the presentation of data, and the management of medical resources through telemedicine. In this article, we present a mathematical data visualization approach for analyzing pandemic data behaviors, such as exponential growth and deviations using the data related to COVID-19 events. Furthermore, this article will include studies on the implications of the COVID-19 pandemic on finance sector.
\end{abstract}

\section{Introduction}

An old Luganda proverb, "Kaami katono okanyoomera mitala wa mugga," means even a minor thing can have an adverse consequence, if it is not handled in the right way. The current crisis of COVID-19 illustrates the true sense of the above proverb. According to the World Health Organization (WHO), the COVID-19 epidemic has resulted in $162,773,940$ confirmed cases and 3,375,573 deaths as of May 18, 2021; see [1]. The "coronavirus" metamorphosis was biologically challenging to study, causing public concern based on early historical pandemics that claimed people's lives. Contagions caused by coronaviruses may increase the likelihood of mutation, which may lead to the development of new virus variant, such as the severe acute respiratory syndrome coronavirus 2 (SARS-CoV-2), severe acute respiratory syndrome (SARS), and Middle East respiratory syndrome (MERS). SARS-CoV-2 was thought to be the first coronavirus to reach pandemic levels in early March 2020, triggering COVID-19 based on earlier SARS and MERS cases; see [2-5].

Early 2020, the entire globe was involved in finding the antiviral vaccine for COVID-19 to save the global population while keeping all standard operating procedures (SOPs) such as social distancing and mask-wearing is highly and carefully enforced among elderly and children all over the world; see [5-11]. As of $4^{\text {th }}$ January 2021, top pharmaceutical companies such as Pfizer and Moderna confirmed that the current vaccine is $95 \%$ effective based on the current raw data; see [12-14]. 
One of the significant effects of the COVID-19 pandemic on the public is potentially paralyzed health systems and economies, mainly in the middle- and lowincome nations. Globally, COVID-19 disease is a problem that needs to be addressed with excellent economic planning and medical strategies. To better understand pandemic outbreak, the response advancement in technologies, advocacy, and mobilization play a crucial role; see [15]. Figure 1 shades an illustration of COVID-19 infection evaluation virologically on the patient who needs an emergency eye surgery when it is scientifically believed that the COVID-19 virus enters human through the eyes, the nose, and the mouth. The present flow chart details potential steps that can be followed in any pandemic or epidemic concerning the general global health responses.

However, the general population adopted all possible ways to resist COVID-19 with standard operating procedure (SOP) requirements, such as social distancing and maskwearing, which are strictly enforced worldwide.

The information and technology sector has managed to keep pandemic data up to date. For instance, using social media to promote public health campaigns is very effective. Table 1 demonstrates the worst epidemics in the history of humans; see $[16,17]$.

There are a few threats involved with the awareness of social media about the COVID-19 pandemic, such as fatalities, pandemic diagnosis, and treatment options like COVID-19 vaccines or COVID-19 medicines given to the patients. All these factors physically and mentally created extra fear and nervousness within the public. This resulted in prevalent chaos, dread buying of home stuff, hoarding of essential commodities by the traders, price increase, violence on the streets, discriminations, conspiracy thinking, etc. [18].

Figure 2 shows counties with high population risks where China and the US are identified. Pandemics and epidemics are considered to be threatening the human race repeatedly. However, large technological companies are working hard to reduce the spread of erroneous information. Google, YouTube, Instagram, and Facebook, for example, have worked tirelessly to direct the public to the most up-todate, verifiable information available through the WHO website; see $[19,20]$.

Fifth-generation connectivity technologies and highspeed Internet with its attendant benefits were launched by convergence technologies such as mobile, cloud, and robots; see $[20,21]$. Artificial intelligence, in particular, has shown to have an important role in promoting health, indicating inoculation techniques involving sympathetic viral protein structure [22].

Based on the COVID-19 pandemic available growth rate data, it is quite complex to determine whether SOPs have been practiced. The pandemic grows exotically and exponentially at a critical stage which is extreme for human computation to understand and analyze. Such complexities raise technical questions like how can exponential growth deviation be determined? Where does exponential data growth emanate from? What is the possible implication of such data growth? What and when does it stop? How can strategic population planning and management be done in case of a pandemic like COVID19 in play? Figure 3 illustrates countries with high population risks such as Italy, Sweden, and France, respectively.

Furthermore, it is important to focus on how various countries such as Italy, China, and the United States have dealt with the stress caused by the pandemic outbreak since 2019. Now, the mathematical bottleneck is that it is extremely complex to determine a new trend in exponential distribution, it is important to determine when it is growing to end or otherwise, determine when exponential ends, it simplifies planning and management of pandemic.

The rest of this paper is structured as follows: some literature facts about the COVID-19 virus are presented in Section 2. Section 3 presents the COVID-19 pandemic key technology trends. In Section 4, the data visualization model is presented. In Section 5, the effect of the COVID-19 pandemic on finance is explained and well demonstrated. In Section 6 , the concluding remarks are given.

\section{Related Literature}

In this section, some facts about the COVID-19 are presented.

2.1. Anosmia as a Symptom. Anosmia is the medical terminology for partial or complete loss of smell. Furthermore, symptoms may include high fever, cough, and shortness of breath among the symptoms listed under COVID-19. Anosmia (loss of smell) and worsened sense of taste are the neurological symptoms and one of the most commonly reported indicators of COVID-19 documented by healthcare physicians. For instance, over 29\% of the public that has tested positive in South Korea for the COVID-19 virus are said that they have lost smell. Also, every two out of three COVID-19 infected persons noticed some level of anosmia; see detail in [22-24].

2.2. Babies Getting Seriously Ill. When compared to the adult population on the COVID-19 ill statistics, children aging one year to 5 years are less likely to become severely affected by COVID-19 if they contact the novel COVID19, while infants are at higher risk. For example, over 2,143 children in China, approximately $12 \%$ of sick infants were critically ill compared to $8 \%$ of children aged one year to 5 years [25-27].

2.3. Nonsymptomatic People Can Spread the Virus. In February 2020, over 565 people from Japan expatriated from Hubei Province, China, are asymptomatic carriers (who positively tested for COVID-19 virus infection never experienced symptoms of COVID-19 [28-30]). Web-based customized applications, such as e-commerce and e-learning sites, provide several strategies for document modeling, 


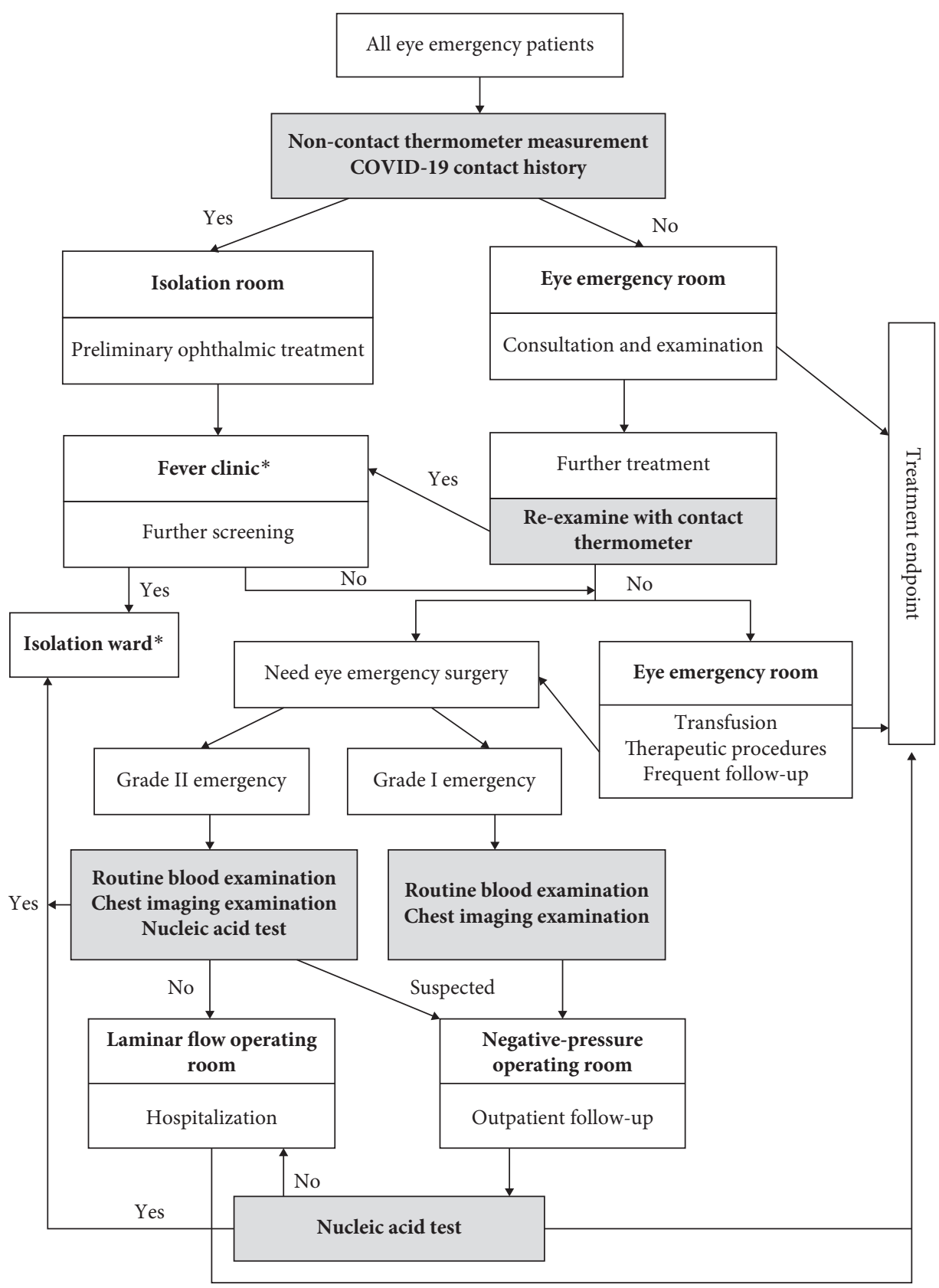

FIGURE 1: COVID-19 infection evaluation on a patient who needs an emergency eye surgery.

knowledge retrieval, and ways to derive information from page content. In such applications, user COVID-19 profiles are typically defined as vectors, with each vector entry representing a weight or degree of interest for each item on the web pages [31, 32].

2.4. Blood Type A Individuals are More Susceptible. Early studies showed that over 2,170 people were hospitalized as a result of the COVID-19 pandemic, suggesting that the number of sick people with blood type A was higher than studies would expect based on the proportion of people with blood type A in the general population, as detailed in $[33,34]$.

\section{Role of Technology in COVID-19 Pandemic}

In this section, the reflection of technology management during COVID-19 pandemic is presented.

3.1. Digital and Contactless Payments. Cash notes can carry the COVID-19 virus to different destinations once an infected person gets access to it. So, central banks have taken various preventive measures in China, the United States of America, and South Korea to warrant notes that are hygienic before they go within the circulation to evade COVID-19 spread [35]. According to the World Bank, more than 1.7 billion unbanked individuals do not have convenient access 
TABLE 1: The worst twelve epidemics in the history of humanity.

\begin{tabular}{lccc}
\hline Fecund & Epidemic name reference & Years of occurrence & Approximated deaths \\
\hline 01 & Plague of Justinian & 541 to 542 & $100,000,000$ \\
02 & Black Plague & 1346 to 1350 & $50,000,000$ \\
03 & HIV/AIDS (human immunodeficiency virus) & 1960 to present & $39,000,000+$ \\
04 & 1918 Flu (Spanish Flu) & 1918 to 1920 & $20,000,000$ \\
05 & Modern Plague & 1894 to 1903 & $10,000,000$ \\
06 & Asian Flu & 1957 to 1958 & $2,000,000$ \\
07 & Sixth Cholera Pandemic & 1899 to 1923 & $1,500,000$ \\
08 & Russian Flu & 1889 to 1890 & $1,000,000$ \\
09 & Hong Kong Flu & 1968 to 1969 & $1,000,000$ \\
10 & Fifth Cholera Pandemic & 1881 to 1896 & 981,899 \\
11 & Ebola & 1976 to 2015 & $13,562+$ \\
12 & 2020: COVID-19 & 2019 to present & $3,375,573+($ as May 18,2021$)$ \\
\hline
\end{tabular}
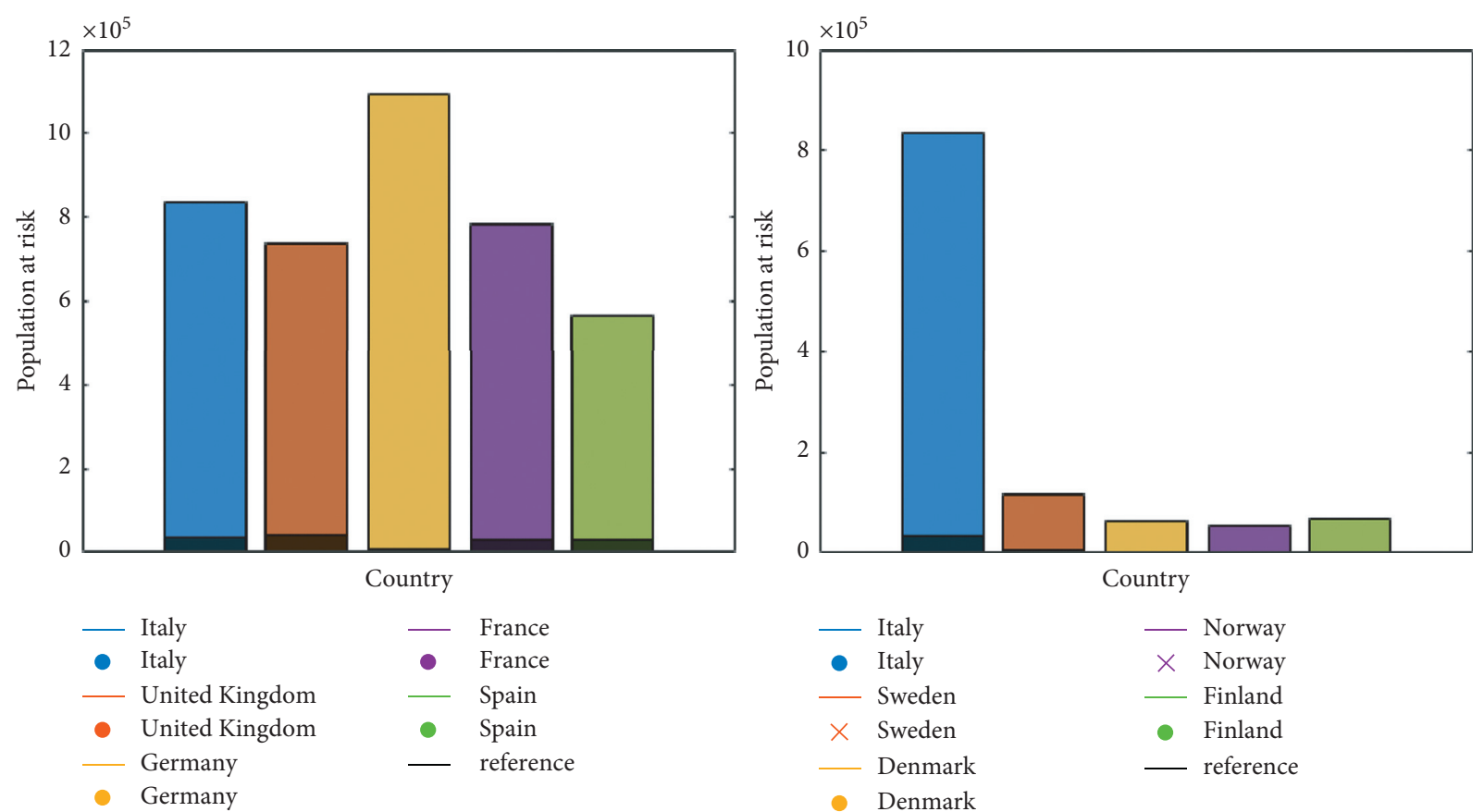

Figure 2: Selected nations with a high population at risk $10 \times 10^{5}$.

to digital payments. The obtainability of digital payments also depends on the availability of the internet, computers, and a network to turn money into a digital format [36]. In addition, COVID-19 also turned online shopping around the globe. Several delivery restaurants and companies within China and the USA are launching numerous delivery units and services where products are hand-picked and dropped off at a selected position as an alternative human-to-human interaction. Such online purchasing necessitates the use of a robust logistical system [37].

3.2. Remote Work. Cloud technologies and work communication software such as Zoom and Skype allow a person to appear in front of a virtual backdrop while remaining in the privacy of their own home. As a result, there will be less movement and less communal transmission; see [38, 39]. In addition to preventing the propagation of COVID-19 infections, remote work saves time and allows for greater flexibility. Employers can choose to cut lease expenses and attract employees from counties with low labor expenses if remote work becomes more popular following the COVID19 epidemic; see [40-42].

3.3. Supply Chain. The COVID-19 has altered the global supply chain dramatically. Distancing and quarantine regulations have forced the closure of several factories. Although food demand and medical and personal protective equipment demand are increasing, certain countries have put varying levels of export bans on these products [41-43].

\section{Data Visualization Model (Methodology)}

In this section, we will describe the mathematical presentation of the proposed mathematical data visualization model (MDVM) for all countries with exponential data growth, depicting the change in data growth incredibly well. 

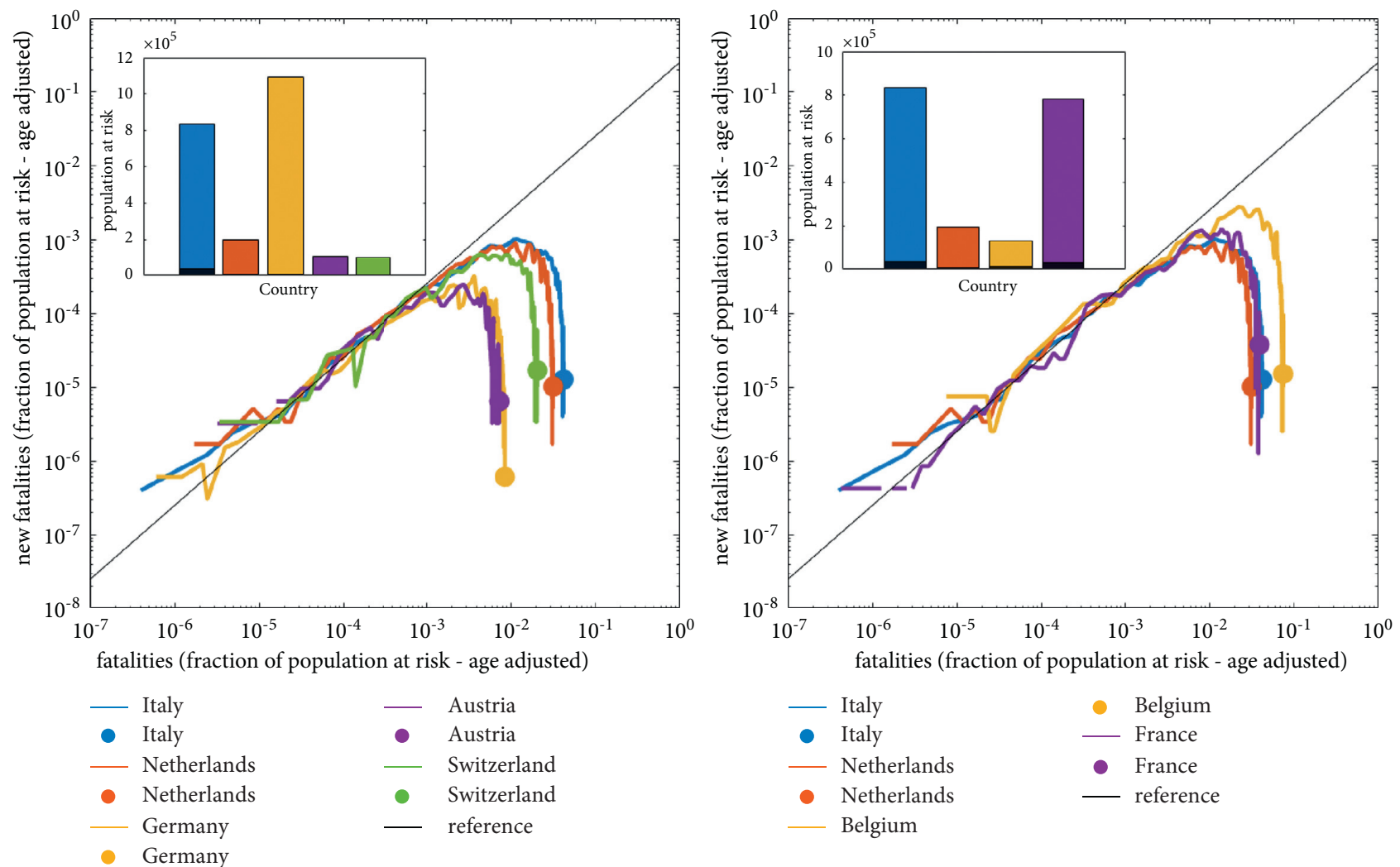

Figure 3: Nations with a high population at risk $10 \times 10^{5}$.

A graph of total confirmed cases and confirmed cases within the last week of pandemic growth appears for nations with a decline in exponential growth. For the graphical illustration, three factors are considered: (a) using a logarithmic scale; it manages exponential growth evidenced by labeling the graphs, making scaling appropriate; (b) focuses on the change, not fundamentally absolute number. Considering cases in South Korea early this year $24^{\text {th }}$ of March 2020 , the total number of cases first was exponential from ten to forty days and beyond that changed the pattern per the total cases, so without graphing, it is quite hard to notice the change in exponential growth; and (c) plot against time, based on physical timing, to cater for exponential; the graphs are plotted against time.

In this situation, the overall population infected with COVID-19 will be $\hbar$, and the current growth rate will be $\theta$. Therefore, we have $\mathrm{d} \hbar / \mathrm{d} t \propto \hbar, \mathrm{d} \hbar / \mathrm{d} t \propto \theta \hbar$,

$$
\frac{\mathrm{d} \hbar}{\mathrm{d} t} \propto \theta \mathrm{d} t
$$

For simplicity,

$\int_{\hbar_{0}}^{\hbar} \frac{\mathrm{d} \hbar}{\hbar}=\int_{0}^{t} \theta \mathrm{d} t,[\ln \hbar]_{\hbar_{0}}^{\hbar}=[\theta t]_{0}^{t}, \ln \hbar-\ln \hbar_{0}, \ln \frac{\hbar}{\hbar_{0}}=\theta t$.

The maximum likelihood approach is used to estimate (1) the prevalence of COVID-19 in the public for various age groups.

$$
\begin{aligned}
\exp \left\{\ln \frac{\hbar}{\hbar_{0}}\right\} & =\exp \{\theta t\}, \\
\hbar & =\hbar_{0} e^{\theta t} .
\end{aligned}
$$

Given a public age and the prevalence risk of various age groups, the incidence of COVID-19 with age-specific group where lambda is age and prevalence is alpha is given as follows:

$$
P(\lambda \mid \alpha)=\frac{P(\lambda \mid \alpha) P(\lambda)}{\sum_{i} P(\alpha \mid \lambda)},
$$

where $P(\lambda \mid \alpha)$ is the prevalence risk of the age group (i); $P$ $\left(\lambda_{i}\right)$ is relative to the age group $(i)$ in the general public.

Let us consider the $\alpha$ different age group is attained by a logistic function of age $\lambda$ as

$$
P(\alpha \mid \lambda)=\frac{1}{[1+\exp (\mu-i / r)]} .
$$

To determine the mortality risk of a pandemic, employ a maximum livelihood methodology that is more connected and articulated when estimating illness mortality.

$$
P\left(\lambda_{i} \mid \phi, \xi\right)=\frac{P(\xi \mid \phi, \lambda) P\left(\lambda_{i} \mid \phi\right)}{\sum_{i} P\left(\xi, \lambda_{i} \mid \phi\right)} .
$$

Given that $\phi$ is the infection, $\xi$ is the mortality rate. Therefore, $P\left(\xi, \phi \mid \lambda_{i}\right)$ is the risk of mortality condition on the individual's age and infection state; $P\left(\lambda_{i} \mid \phi, \xi\right)$ becomes the age composition of fatal cases of COVID-19 pandemic. We consider that infection happens in all age groups for the general public and therefore express. We further applied the 
maximum likelihood approach to attain the mortality risk of COVID-19 for the diverse age groups in public. Moreover, there was an imputation of the mortality rate of the infected people, thus

$$
\begin{aligned}
P\left(\lambda_{i} \mid \phi\right) & =P\left(\lambda_{i}\right), \\
P(\xi \mid \phi) & =\sum_{i} P\left(\xi, \lambda_{i} \mid \phi\right) .
\end{aligned}
$$

At first, as shown in Figure 4, scaling a thousand and ten thousand appears to be close to each other. Nevertheless, the log-scale on the $X$-axis makes it necessary to determine SOPs implementation; so, applying linear trends is better to display the pattern of data growth. Second, time is inferred; unlike any other COVID-19 graphing that we have seen, time is not on the $Y$-axis, which may be configuring error and confusing, but time is shown through animation. Third, when comparing confirmed cases to infections, the total confirmed subjects are shown rather than the exact number of cases. It is unknown how many COVID-19 positive cases exist; however, the screening test for COVID-19 indicates a trend in the spread of infection. Assuming that the data are accurate, the countries that appear below the graph depicted a usual trend that desired all countries to reduce the pandemic spread and growth. Daily news and reports mainly focus on current data, yet the understanding trend is significant for planning and management. Assuming the data are correct, the countries displayed below the graph show a typical pattern that is desirable for all countries to reduce pandemic transmission and expansion. While daily news and reports primarily focus on current data, understanding trend is critical for planning and management.

Visual representation of the provided data, such as growth rate versus tested cases, reveals some inconsistencies in that set of data. It is also important to note that pandemic such as COVID-19 and others spread more slowly in real life than in the graph, but the data used not only show a number of cases but also a number of tests, causing the confirmed case to grow faster. Because of fundamental data errors in health facilities around the world, the used data are obtained from incomplete data sources. Further, each country is capable of conducting COVID-19 testing facilities differently. The charts for the latest weeks were created by tracking the pattern/trend for a few days, which causes data inconsistency due to daily growth rates; see Figure 5.

Data for every country is computed using a logarithmic scale when existing cases have been plotted against new cases. It has been observed that disease spreading is uncontrolled with exponential growth rate. However, strict application of SOPs may lead to reduction in exponential growth rate. Moreover, results also justify the validity of the proposed model; see Figure 6.

\section{The Effect of COVID-19 on Finance Sector}

In this section, we determine the impact of COVID-19 on finance. For this purpose, a case study of the performance of the Pakistani stock market is considered. In this study, we use the data of COVID-19 daily cases and the closing prices of PSX (Pakistan Stock Exchange), 100 indexes of February and March 2021.

5.1. Research Methodology. In this subsection, we discuss the research model which we used to determine the impact of COVID-19 on the performance of the Pakistani stock market. The model of this study can be expressed as

$$
\text { Prices }_{\text {it }}=\beta_{0}+\beta_{1} \text { Cases }_{\text {it }}+\varepsilon_{\text {it }} \text {, }
$$

where Prices $_{i t}$ represents the daily closing price of PSX; $\beta_{0}$ is the intercept of the model; Cases $_{\text {it }}$ denotes the COVID-19 daily cases; $\epsilon_{\mathrm{it}}$ indicates the error term. It is important to note that Prices $_{\text {it }}$ is the response variable of the model and it is measured by the daily prices of PSX, and Cases ${ }_{i t}$ is the predictor variable of the model and it is measured by the frequency of the COVID-19 daily cases in Pakistan.

5.2. Data and Resources. The data used in this study are obtained from two different sources. The data of the response variable are obtained from the PSX data portals. These data can be retried from the following link: https://dps. psx.com.pk/monthly-reports. However, the data of the predictor variable is available at https://www.worldometers. info/coronavirus/country/pakistan/. This behavior of the considered datasets is displayed in Figure 7, and the summary statistics are provided in Table 2.

5.3. Regression Analysis. In this subsection, we consider a linear regression technique to model the relationship

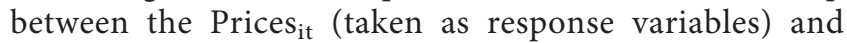
Cases $_{\text {it }}$ (taken as predictor variables). After applying the regression technique, we observe that $\beta_{0}=46469.1719$, interpreted as the predicted stock market price in the absence of COVID-19 influences. The slope (regression coefficient) of the regression model provided in (1) is $\beta_{1}=-0.4434$, indicating -0.4434 units decrement in the stock market prices. Therefore, we conclude that the COVID-19 daily cases have a negative relationship with the stock market prices. Thus, the fitted regression model is given by

$$
\text { Prices }_{\text {it }}=46469.1719-0.4434 \text { Cases }_{\mathrm{it}} .
$$

The relationship between stock market prices and daily COVID-19 cases is displayed graphically in Figure 8. The plot is presented in Figure 8, indicating a negative relationship between Prices $_{i t}$ and Cases ${ }_{i t}$.

5.4. Hypothesis Testing. To check the significance of the COVID-19 daily cases on stock market prices, a statistical technique called hypothesis testing is adopted. The null and the alternative hypothesis can be formulated as follows: $H_{0}=$ the COVID-19 daily cases have no influence on the Pakistani stock market vs $H_{1}=$ the COVID-19 daily cases have a significant influence on the Pakistani stock market. 

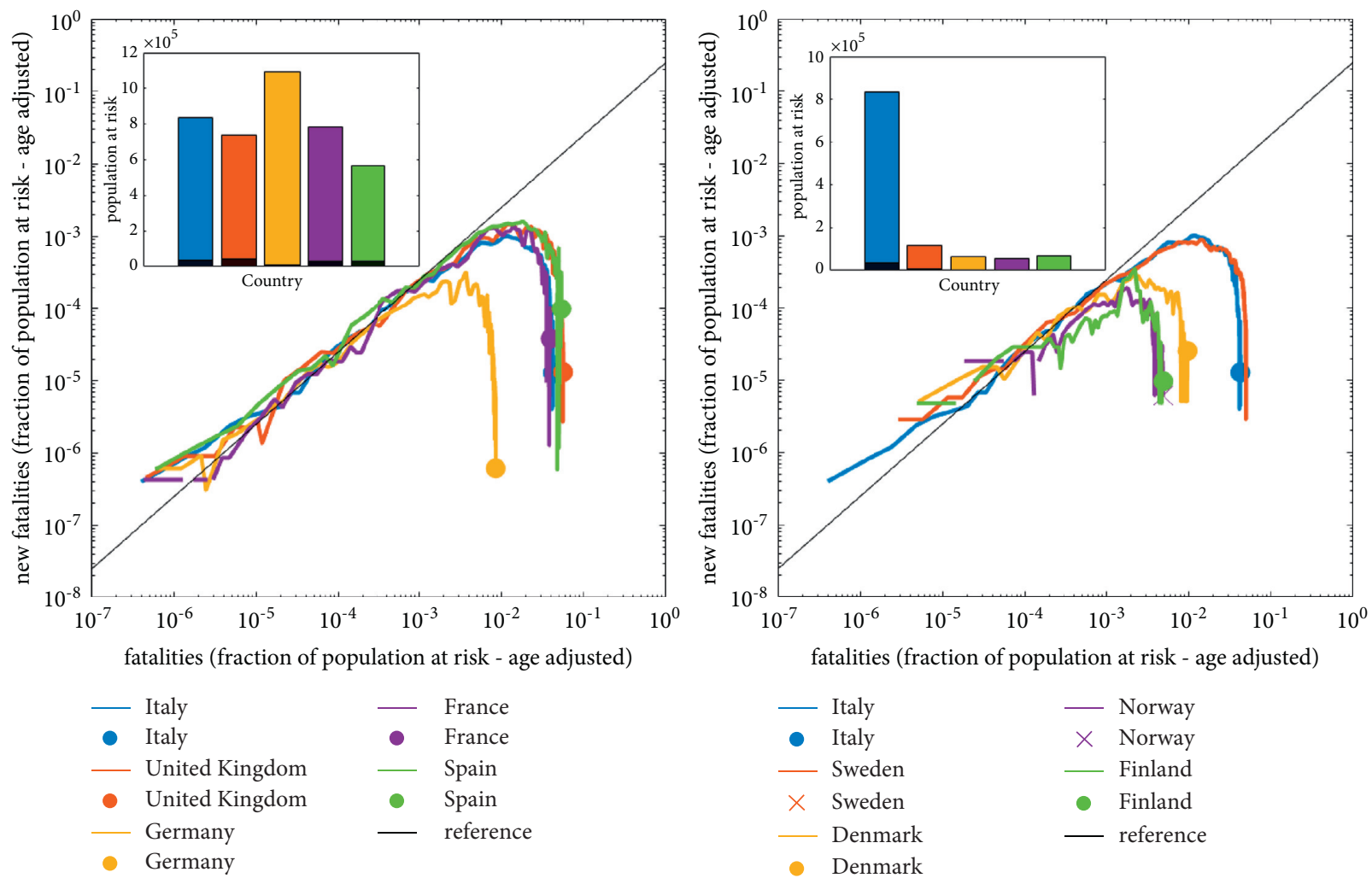

Figure 4: COVID-19 visual deviation representation of high population risk.
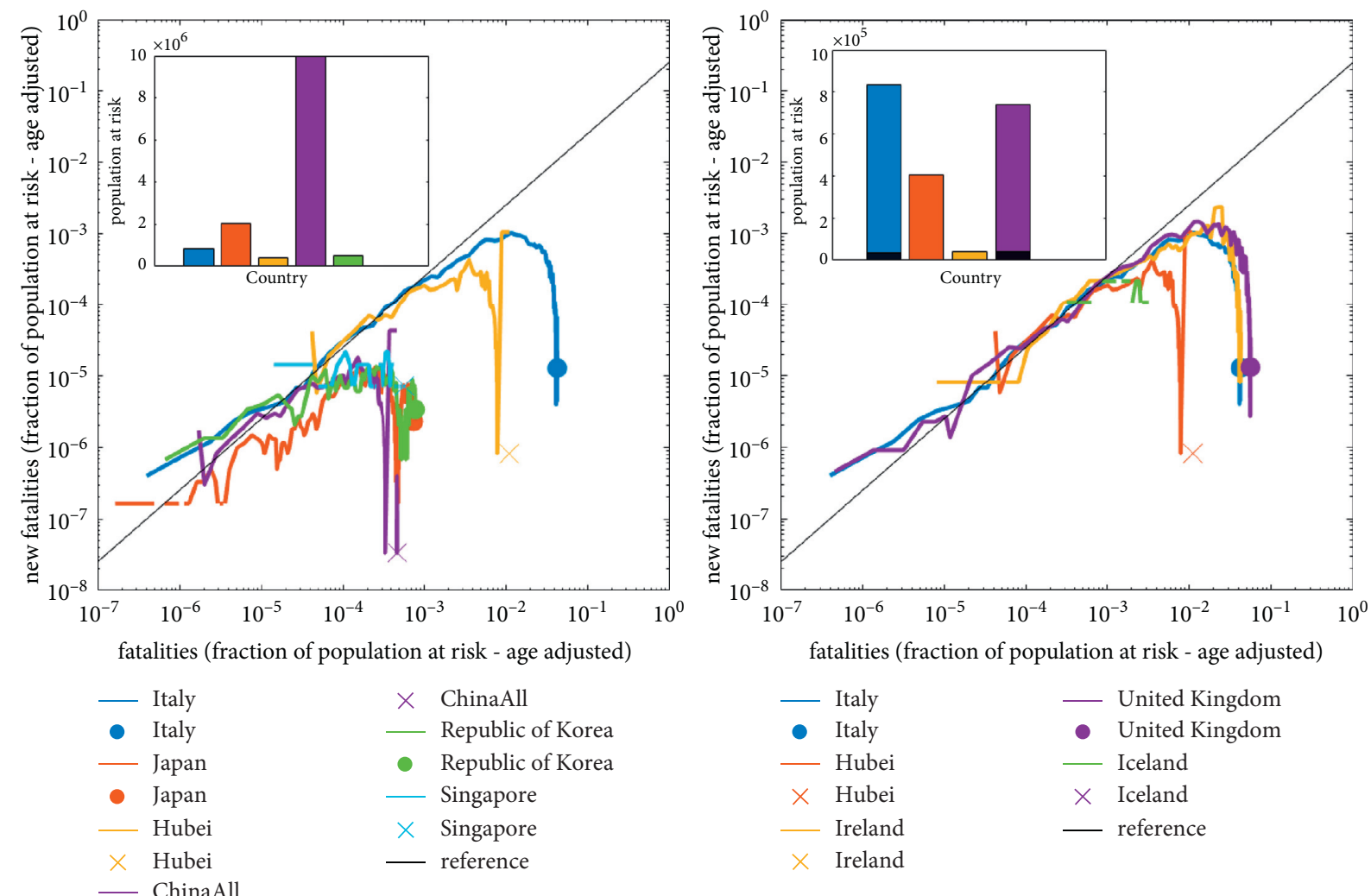

Figure 5: COVID-19 visual deviation representation of medium population risk. 


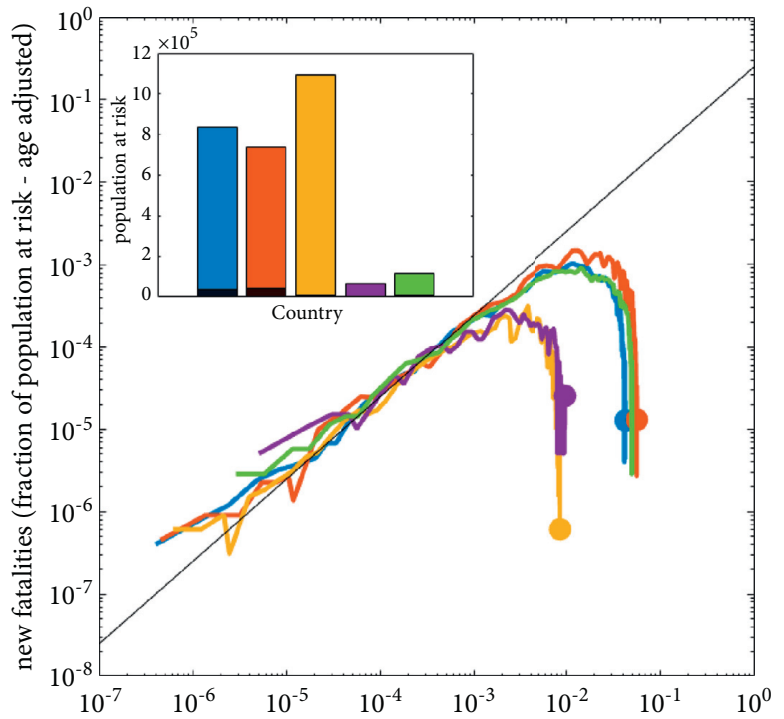

fatalities (fraction of population at risk - age adjusted)
- Italy
- Italy
- United Kingdom
- United Kingdom
- Germany
- Germany

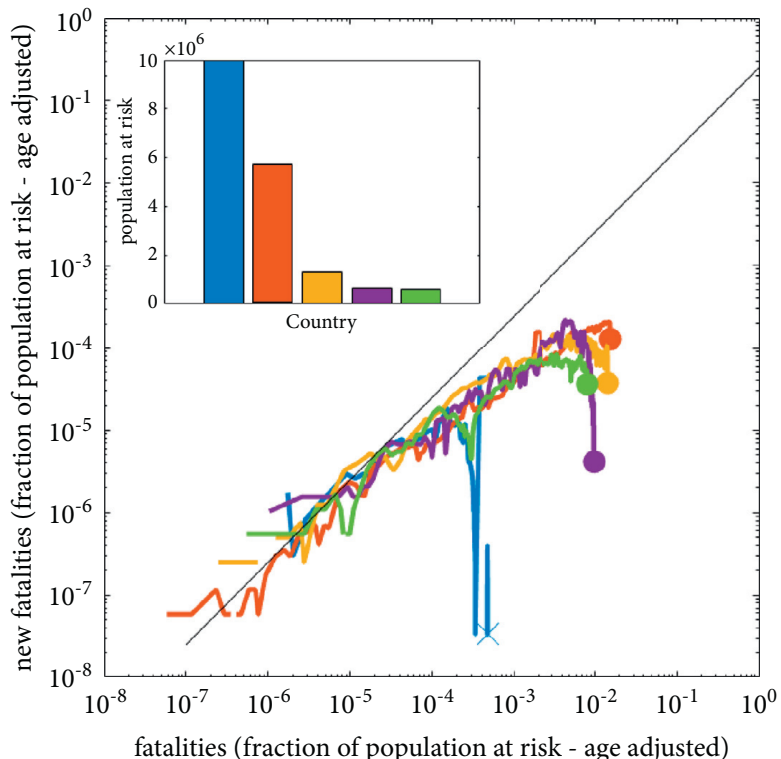

\begin{tabular}{|c|c|c|}
\hline ChinaAll & 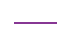 & Pakistan \\
\hline ChinaAll & $\bullet$ & Pakistan \\
\hline India & & Bangladesh \\
\hline India & - & Bangladesh \\
\hline Russia & 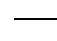 & reference \\
\hline
\end{tabular}

Figure 6: COVID-19 visual deviation representation of medium population risk.

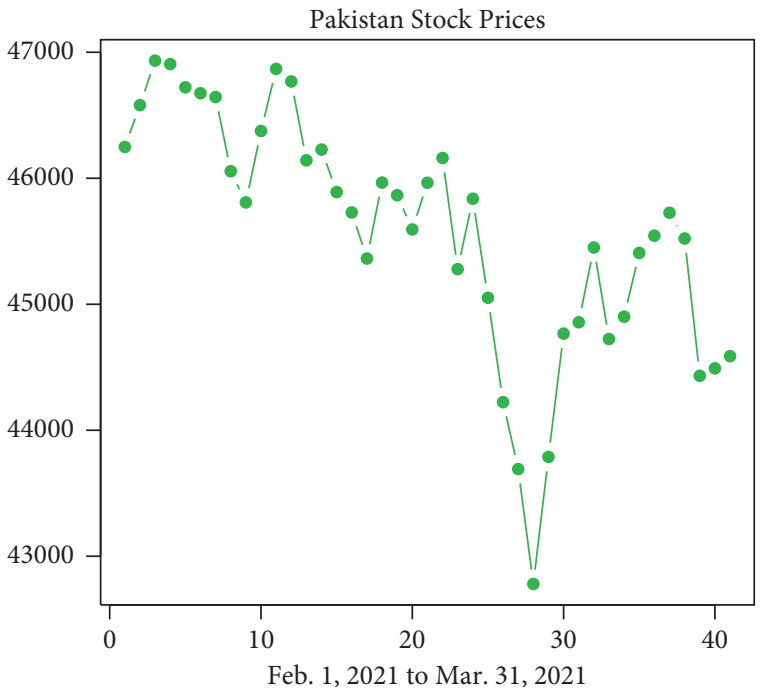

- Stock Prices

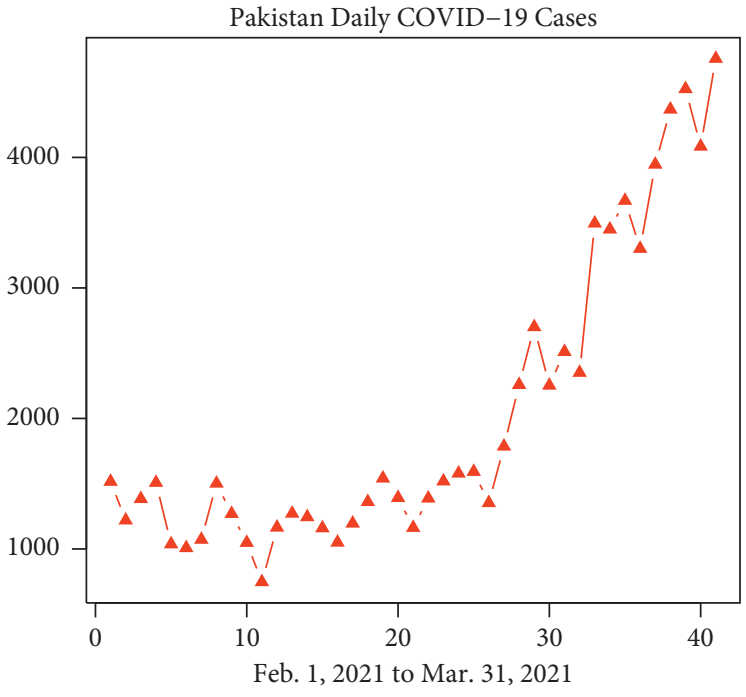

A Daily COVID-19 Cases

FIGURE 7: Graphical display of the responsible and predictor variables.

TABLE 2: Summary measures of the data based on the responsible and predictor variables.

\begin{tabular}{lcccccc}
\hline Variables & Min. & 1st qu. & Median & Mean & 3rd qu. & Max. \\
\hline Prices $_{\text {it }}$ & 42779.76 & 44901.31 & 45728.75 & 45574.25 & 927628.90 & 46933.63 \\
Cases $_{\text {it }}$ & 747 & 1220 & 1508 & 2018 & 2511 & 4757 \\
\hline
\end{tabular}




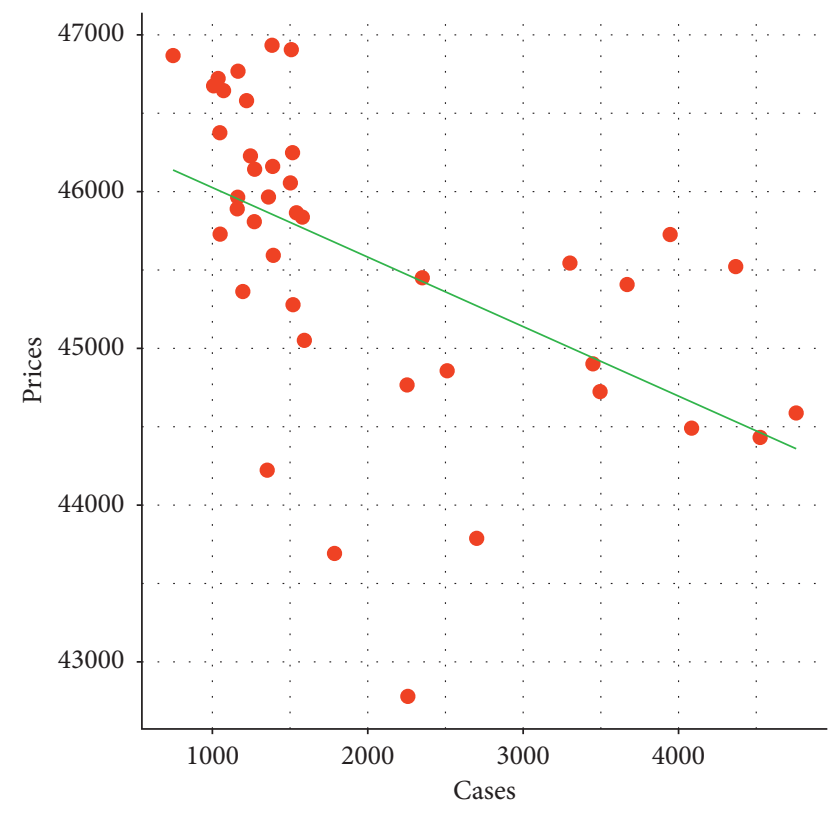

FIGURE 8: Relationship between stock market prices and COVID-19 cases.

The standard errors are very useful in carrying out hypothesis tests on the regression coefficients. In this case, it measures how much the coefficient estimates differ from the average value of Prices ${ }_{i t}$. The standard error also plays a prominent role in calculating the confidence intervals. If the standard errors of the estimates of $\beta_{0}$ and $\beta_{1}$ are sufficiently small, then even small values of the estimates of $\beta_{0}$ and $\beta_{1}$ will provide sufficient evidence against $H_{0}$. Here, we use the $\mathrm{t}$-statistic to measure how far $\beta_{0}$ and $\beta_{1}$ are from zero.

5.5. Statistical Testing. The $t$-statistic value represents how far (in standard deviations) the coefficient estimate is from zero. A large value of the $t$-statistic provides evidence against $H_{0}$, indicating that a relationship exists between Prices ${ }_{i t}$ and Cases ${ }_{i t}$. The smaller the $p$ value, the greater the likelihood of rejecting $H_{0}$. Generally, a $p$ value of 0.05 is standard cut-off point. After applying the $t$-test, the numerical results are provided in Table 3.

From the results provided in Table 2, we can see that the $p$ value is less than 0.05 indicating that the values of $\beta_{0}$ and $\beta_{1}$ are not equal to zero. Therefore, we reject $H_{0}$ and conclude that there is a significant relationship between Prices $_{\text {it }}$ and Cases $_{\text {it. }}$.

The F-test statistic is another prominent tool used to detect the relationship between response and predictor variables. After applying the $F$-test, we observe that the $p$ value is 0.0004147 and the $F$-statistic is 14.9. $p$ value less than 0.05 indicates a significant influence of Cases ${ }_{\text {it }}$ on Prices ${ }_{i t}$.

5.6. Accuracy of the Model Fitting. The $R$ square $R^{2}$ is the most important quantity to measure regression model fit, and its value varies between 0 and 1 . It quantifies the linear relationship between predictor and response variables. The value of $R^{2}$ is close to 1 representing the better fit and close to 0 representing the poor fit. In our case, the $R^{2}$ is 0.2765 , which means only $0.2765 \%$ of the variation in Prices it is explained by Cases ${ }_{i t}$.

5.7. Residuals. The residual standard error measures the quality of the regression fit. In this study, it represents the difference between the Prices ${ }_{i t}$ and the true regression line. The residuals vs fitted plot of the Cases ${ }_{i t}$ is presented in Figure 9. From the residuals vs fitted plot provided in Figure 5, the red line is almost lying near the residual value of 0 and is almost horizontal and all the fitted values are scattered around them without a systematic relationship. Therefore, we conclude that the residuals are linearly related.

The normality of the residual can be detected via two approaches such as normality test and graphical approach. Under the domain of normality test, we perform the Shapiro-Wilk (SW) normality test and Anderson-Darling (AD) normality test to check the normality of the residuals. Under these two tests, the null and alternative hypothesis can be constructed as follows: $H_{0}=$ the residuals are normally distributed vs $H_{1}=$ the residuals are not normally distributed. After performing the analysis, we observe $\mathrm{SW}=0.89199$ with $p$ value $=0.0009702$ and $\mathrm{AD}=1.2203$ with $p$ value $=0.003085$. As we can see that the $p$ values for both the tests are less than 0.05 , therefore, we reject $H_{0}$ and conclude that the residuals are not normally distributed.

The Q-Q (quantile-quantile) plot, is a graphical approach used to determine whether the data collection is from a specific distribution such as normal, Weibull, or exponential. In fact, a Q-Q plot is a form of the scatter plot created by plotting two sets of quantiles against each other. If both sets of quantiles come from the same distribution, then all the points form a straight line. The Q-Q plot of Prices ${ }_{i t}$ is sketched in Figure 9, which shows the residuals are roughly linear related. Henceforth, we conclude that the normality is hardly met on residuals.

To check the independence of residuals, we use the Durbin-Watson (DW) test. Under the DW test, the $H_{0}$ and $H_{1}$ hypothesis can be constructed as follows: $H_{0}=$ the residuals are not linearly related vs $H_{1}=$ the residuals are linearly related. After applying the DW test, we observe that $\mathrm{DW}=0.8141324$ with $p$ value $=0.3622015$. As the $p$ value is greater than 0.05 , therefore, we fail to reject the $H_{0}$ and conclude that the residuals are normally related.

Homoscedasticity is an important assumption in the regression analysis which states that the variance of the residuals is approximately constant for all values of the predictor variables. Violation of this assumption results in a large variance. From the plot scale-location plots provided in Figure 9, we can see that all the residuals are scattered leading to the fact the homoscedasticity met on the residuals.

5.8. Influential Observations. The influential observations play a significant role in statistical analysis. In particular, an influential observation is one whose deletion has a significant effect on the estimates. The traditional cut-off of $4 / n$ is used to identify the influential observations. The numbers 
TABle 3: Summary measures of the economics data.

\begin{tabular}{lccccc}
\hline Variable & Coefficient & Estimated value & Standard error & $t$-statistics & $p$ value \\
\hline \multirow{2}{*}{ Cases $_{\text {it }}$} & $\beta_{0}$ & 46469.1719 & 265.5734 & 174.98 & $2 e-16$ \\
& $\beta_{1}$ & -0.4434 & 0.1149 & -3.86 & 0.000415 \\
\hline
\end{tabular}

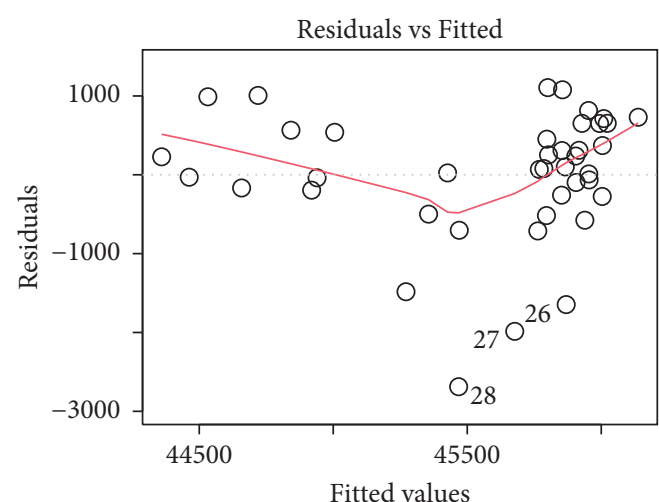

(a)

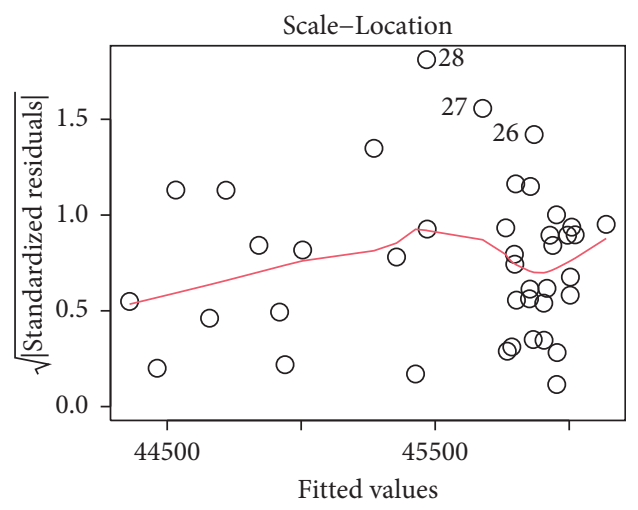

(c)

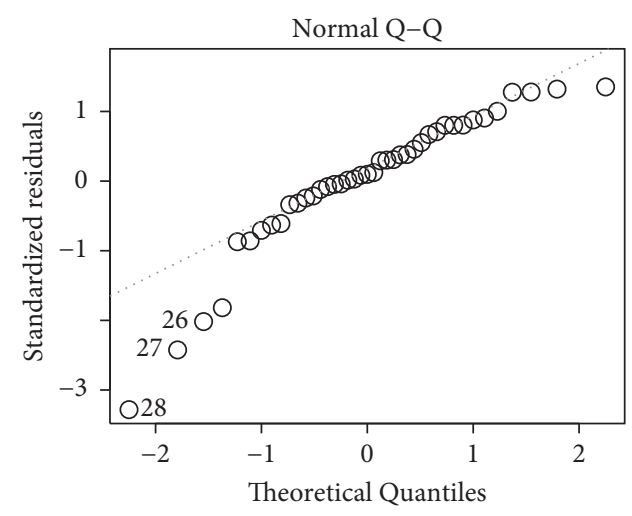

(b)

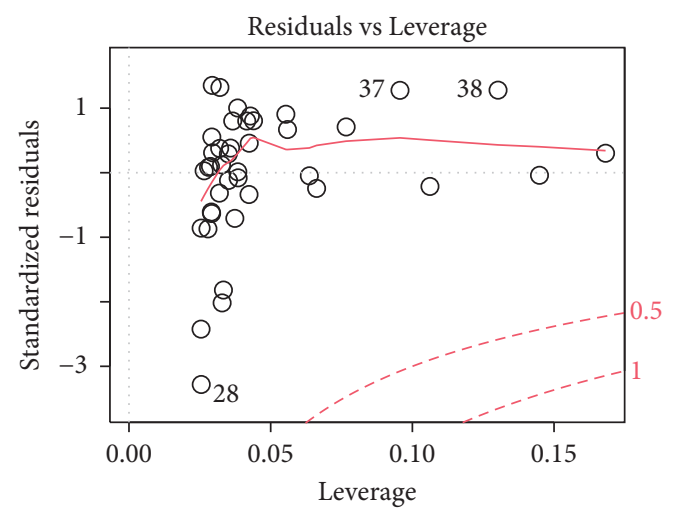

- - - Cook's distance

(d)

FIgUre 9: Plots of (a) residuals vs fitted, (b) normal Q-Q, (c) scale-location, and (d) residuals vs leverage.

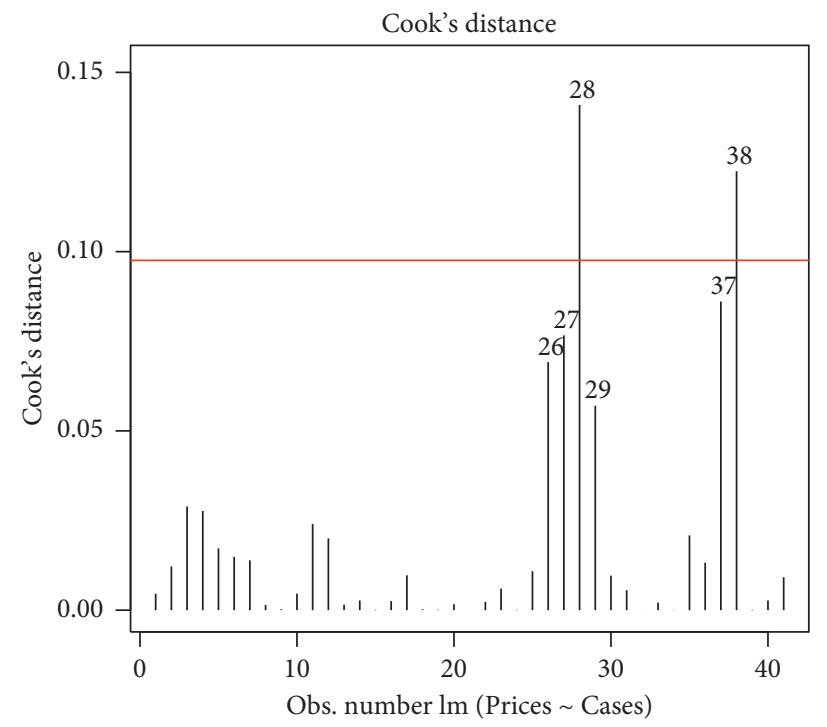

FIGURE 10: The plot of influential observations using Cook's distance approach. 
which are crossing Cook's distance line representing the influence observations. A graphical display of the influential observations using the Cook's distance approach is provided in Figure 10.

\section{Concluding Remarks}

This paper demonstrated COVID-19's effect on finance and also depicted technical roles in managing the COVID-19 pandemic. The outbreak of COVID-19 has negatively impacted finance and health sectors. In this article, we suggested a mathematical data visualization model that analyzes pandemic data behaviors such as exponential growth and deviations. It is noted that the trends of infected cases, recoveries, and deaths have their assumptions and parameters varying from one regional demographic territory to another. Finally, a regression tool is adopted to see the impact of the COVID-19 pandemic on finance sector. According to the findings of this study, it is observed that the COVID-19 pandemic has negative impact on finance sector.

\section{Data Availability}

All the links of the datasets used in this paper are provided within the main body of the manuscript.

\section{Conflicts of Interest}

The authors declare that they have no conflicts of interest.

\section{Acknowledgments}

This work was funded by the Department of Statistics, Yazd University, Iran.

\section{References}

[1] World Health Organization, WHO Coronavirus Disease (COVID-19) Dashboard, https://covid19.who.int/.

[2] J. Heer, M. Bostock, and V. Ogievetsky, "A tour through the visualization zoo," Communications of the ACM, vol. 53, no. 6 , pp. 59-67, 2010.

[3] J. P. Ehrenberg, J. Utzinger, G. Fontes et al., "Efforts to mitigate the economic impact of the COVID-19 pandemic: potential entry points for neglected tropical diseases," Infectious diseases of poverty, vol. 10, no. 1, pp. 2-10, 2021.

[4] D. Miconi, Z. Y. Li, R. L. Frounfelker et al., "Ethno-cultural disparities in mental health during the COVID-19 pandemic: a cross-sectional study on the impact of exposure to the virus and COVID-19-related discrimination and stigma on mental health across ethno-cultural groups in Quebec (Canada)," BJPsych Open, vol. 7, no. 1, 2021.

[5] N. Zhao and F. You, "Food-energy-water-waste nexus systems optimization for New York State under the COVID-19 pandemic to alleviate health and environmental concerns," Applied Energy, vol. 282, Article ID 116181, 2021.

[6] A. E. Boutzoukas, I. C. Akinboyo, C. A. Wong, D. K. Benjamin, and K. O. Zimmerman, "Impact of COVID19-related school closures on the drivers of child health," North Carolina Medical Journal, vol. 82, no. 1, pp. 50-56, 2021.
[7] M. Ghahremanloo, Y. Lops, Y. Choi, and S. Mousavinezhad, "Impact of the COVID-19 outbreak on air pollution levels in East Asia," The Science of the Total Environment, vol. 754, Article ID 142226, 2021.

[8] M. Rathi, P. Singh, H. P. Bi et al., "Impact of the COVID-19 pandemic on patients with systemic lupus erythematosus: observations from an Indian inception cohort," Lupus, vol. 30, no. 1, pp. 158-164, 2021.

[9] R. P. Rannan-Eliya, N. Wijemunige, J. R. N. A. Gunawardana et al., "Increased Intensity of PCR Testing Reduced COVID19 transmission within countries during the first pandemic wave: study examines increased intensity of reverse transcriptionpolymerase chain reaction (PCR) testing and its impact on COVID-19 transmission," Health Affairs, vol. 40, no. 1, pp. 70-81, 2021.

[10] P. K. Narayan, Q. Gong, and H. J. A. Ahmed, "Is there a pattern in how COVID-19 has affected Australia's stock returns?" Applied Economics Letters, pp. 1-4, 2021.

[11] J. M. Rhodes, S. Subramanian, E. Laird, G. Griffin, and R. A. Kenny, "Perspective: vitamin D deficiency and COVID19 severity-plausibly linked by latitude, ethnicity, impacts on cytokines, ACE2 and thrombosis," Journal of Internal Medicine, vol. 289, no. 1, pp. 97-115, 2021.

[12] D. Karunanidhi, P. Aravinthasamy, M. Deepali, T. Subramani, and K. Shankar, "Groundwater pollution and human health risks in an industrialized region of southern India: impacts of the COVID-19 lockdown and the monsoon seasonal cycles," Archives of Environmental Contamination and Toxicology, vol. 80, no. 1, pp. 259-276, 2021.

[13] E. Ikiz, V. W. Maclaren, E. Alfred, and S. Sivanesan, "Impact of COVID-19 on household waste flows, diversion and reuse: the case of multi-residential buildings in Toronto, Canada," Resources, Conservation and Recycling, vol. 164, Article ID 105111, 2021.

[14] W. E. Copeland, E. McGinnis, Y. Bai et al., "Impact of COVID-19 pandemic on college student mental health and wellness," Journal of the American Academy of Child \& Adolescent Psychiatry, vol. 60, no. 1, pp. 134-141, 2021.

[15] E. A. N. Wastnedge, R. M. Reynolds, S. R. van Boeckel et al., "Pregnancy and COVID-19," Physiological Reviews, vol. 101, no. 1, pp. 303-318, 2021.

[16] T. Day, I.-C. C. Chang, C. K. L. Chung, W. E. Doolittle, J. Housel, and P. N. McDaniel, "The immediate impact of COVID-19 on postsecondary teaching and learning," The Professional Geographer, vol. 73, no. 1, pp. 1-13, 2021.

[17] I. Anil and O. Alagha, "The impact of COVID-19 lockdown on the air quality of Eastern Province, Saudi Arabia," Air Quality, Atmosphere \& Health, vol. 14, no. 1, pp. 117-128, 2021.

[18] J. Iwanaga, M. Loukas, A. S. Dumont, and R. S. Tubbs, “A review of anatomy education during and after the COVID-19 pandemic: revisiting traditional and modern methods to achieve future innovation," Clinical Anatomy, vol. 34, no. 1, pp. 108-114, 2021.

[19] D. Wang, S. Marmo-Roman, K. Krase, and L. Phanord, "Compliance with preventative measures during the COVID19 pandemic in the USA and Canada: results from an online survey," Social Work in Health Care, pp. 1-16, 2021.

[20] R. C. Urban and L. Y. K. Nakada, "COVID-19 pandemic: solid waste and environmental impacts in Brazil," The Science of the Total Environment, vol. 755, Article ID 142471, 2021.

[21] I. Djekic, A. Nikolić, M. Uzunović et al., "Covid-19 pandemic effects on food safety-multi-country survey study," Food Control, vol. 122, Article ID 107800, 2021. 
[22] J. Martorell-Marugn, J. A. Villatoro-Garca, A. Garca-Moreno et al., "DatAC: a visual analytics platform to explore climate and air quality indicators associated with the COVID-19 pandemic in Spain," The Science of the Total Environment, vol. 750, Article ID 141424, 2021.

[23] M. Naeem, "Do social media platforms develop consumer panic buying during the fear of COVID-19 pandemic," Journal of Retailing and Consumer Services, vol. 58, Article ID 102226, 2021.

[24] M. L. Bell and K. C. Fong, "Gender differences in first and corresponding authorship in public health research submissions during the COVID-19 pandemic," American Journal of Public Health, vol. 111, no. 1, pp. 159-163, 2021.

[25] M. Talwar, S. Talwar, P. Kaur, N. Tripathy, and A. Dhir, "Has financial attitude impacted the trading activity of retail investors during the COVID-19 pandemic?" Journal of Retailing and Consumer Services, vol. 58, Article ID 102341, 2021.

[26] C. Ruiz-Frutos, M. Ortega-Moreno, R. Allande-Cussó, S. Domínguez-Salas, A. Dias, and J. Gómez-Salgado, "Healthrelated factors of psychological distress during the COVID-19 pandemic among non-health workers in Spain," Safety Science, vol. 133, Article ID 104996, 2021.

[27] U. Mandelkorn, S. Genzer, S. Choshen-Hillel et al., "Escalation of sleep disturbances amid the COVID-19 pandemic: a cross-sectional international study," Journal of Clinical Sleep Medicine, vol. 17, no. 1, pp. 45-53, 2021.

[28] P. N. Halkitis, "Managing the COVID-19 pandemic: biopsychosocial lessons gleaned from the AIDS epidemic," Journal of Public Health Management and Practice, vol. 27, no. 1, pp. S39-S42, 2021.

[29] P. R. Burton, N. P. Morris, and M. E. Hirschtritt, "Mental health services in a US prison during the CoViD-19 Pandemic," Psychiatric Services, vol. 72, no. 4, pp. 458-460, 2021.

[30] N. Kravitz-Wirtz, A. Aubel, J. Schleimer, R. Pallin, and G. Wintemute, "Public concern about violence, firearms, and the COVID-19 pandemic in California," JAMA Network Open, vol. 4, no. 1, Article ID e2033484, 2021.

[31] E. G. Lewis, M. Breckons, R. P. Lee, C. Dotchin, and R. Walker, "Rationing care by frailty during the COVID-19 pandemic," Age and Ageing, vol. 50, no. 1, pp. 7-10, 2021.

[32] Q. Yuan, B. Qi, D. Hu et al., "Spatiotemporal variations and reduction of air pollutants during the COVID-19 pandemic in a megacity of Yangtze River Delta in China," The Science of the Total Environment, vol. 751, Article ID 141820, 2021.

[33] G. Lambert, K. Drummond, V. Ferreira, and F. Carli, “Teleprehabilitation during COVID-19 pandemic: the essentials of "what" and "how"' Supportive Care in Cancer, vol. 29, no. 2, pp. 551-554, 2021.

[34] Y. F. Liu, Z. Zhang, X. L. Pan et al., "The chronic kidney disease and acute kidney injury involvement in COVID-19 pandemic: a systematic review and meta-analysis," PLoS One, vol. 16, no. 1, Article ID e0244779, 2021.

[35] S. L. Greer, A. de Ruijter, and E. Brooks, "The COVID-19 pandemic: failing forward in public health," in The Palgrave Handbook of EU Crises, pp. 747-764, Palgrave Macmillan, Cham, Switzerland, 2021.

[36] E. Portacolone, A. Chodos, J. Halpern et al., "The effects of the COVID-19 pandemic on the lived experience of diverse older adults living alone with cognitive impairment," The Gerontologist, vol. 61, no. 2, pp. 251-261, 2021.

[37] P. R. Pietromonaco and N. C. Overall, "Applying relationship science to evaluate how the COVID-19 pandemic may impact couples relationships," American Psychologist, vol. 76, no. 3, pp. $438-450,2020$.
[38] N. W. Ruktanonchai, J. R. Floyd, S. Lai et al., “Assessing the impact of coordinated COVID-19 exit strategies across Europe," Science, vol. 369, no. 6510, pp. 1465-1470, 2020.

[39] S. L. Klein, S. Dhakal, R. L. Ursin, S. Deshpande, K. Sandberg, and F. Mauvais-Jarvis, "Biological sex impacts COVID-19 outcomes," PLoS Pathogens, vol. 16, no. 6, Article ID e1008570, 2020.

[40] L. Di Domenico, G. Pullano, C. E. Sabbatini, P. Y. Bolle, and V. Colizza, "Impact of lockdown on COVID-19 epidemic in le-de-France and possible exit strategies," BMC Medicine, vol. 18, no. 1, pp. 1-13, 2020.

[41] G. Corbie-Smith, M. K. Wolfe, S. M. Hoover, and G. Dave, "Centering equity and community in the recovery of the COVID-19 pandemic," North Carolina Medical Journal, vol. 82, no. 1, pp. 62-67, 2021.

[42] C. M. Barkoff and S. A. Mousa, "Pharmacotherapy in COVID 19: potential impact of targeting the complement system," Biomedicines, vol. 9, no. 1, p. 11, 2021.

[43] P. Jiang, X. Fu, Y. V. Fan et al., "Spatial-temporal potential exposure risk analytics and urban sustainability impacts related to COVID-19 mitigation: a perspective from car mobility behaviour," Journal of Cleaner Production, vol. 279, Article ID 123673, 2021. 